\title{
Performance Comparison of Blind Equalization Algorithm
}

\author{
Wenjun $\mathrm{Su}^{1, \mathrm{a}^{*}}$ and Haitao Chen ${ }^{1, \mathrm{~b}}$ \\ ${ }^{1}$ Department of Electronic Information, Guangzhou Civil Aviation College \\ Guangzhou 510000, China

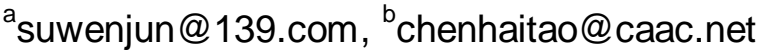 \\ *The corresponding author
}

Keywords: CMA; BSE; FSE

\begin{abstract}
Theory and method of Baud spaced equalizer (BSE) and fractionally spaced equalizer (FSE) based on constant modulus algorithm (CMA) are reviewed. For the un-changed characteristics channel, adaptive constant modulus algorithm can achieve good performance in blind equalization. But the tracking ability of adaptive constant modulus algorithm gets worse while the char-acteristics of channel changes more quickly. Fractional-ly spaced equalizer can achieve distortion compensation in the received signal and better performance than baud-spaced equalizer, because aliasing caused by under sampling is avoided.
\end{abstract}

\section{Introduction}

In wireless communication, the information source is mapped to a character, which is valued in a finite char-acter set. so that it is a non-Gauss signal. Before they are received by one or more sensors, they need to be transmitted through the channel, where they will be affected by intersymbol interference (ISI) and inter channel interference (ICI). The receiver noise is narrow, so it is often modeled as additive Gauss noise. Inter-symbol interference and inter channel interference main-ly due to limited band transmitting and receiving filters, amplifiers, delay and multipath transmission, the rela-tive motion between the transmitter and receiver, cou-pling effect and multiple access interference.

In the case of known channel characteristics $\mathrm{C}$ (f), it is not difficult to design the optimal demodulation filter for the received signal. The problem is that the channel characteristic $\mathrm{C}(\mathrm{f})$ is often unknown in practice. And the response of the mobile radio channel is time variant. For such channels, we cannot design a fixed optimal demodulation filter.

The basic method to solve the inter symbol interference problem is to design the receiver which can compensate or reduce the interference between the received signals. That is the equalizer. Since the channel characteristics are unknown and time-varying, the effective equalizer should be adaptive and change with the change of chan-nel characteristics. The traditional equalization tech-nique uses the pre-defined guidance training to adjust the filter at the receiver. However, this method takes up the channel capacity, and the blind equalization does not need to occupy the bandwidth. Adaptive algorithms are mainly Kalman filtering algorithm, least mean square (LMS) algorithm, recursive least squares (RLS) algorithm and constant modulus (CM) algorithm.

Linear channel equalization can eliminate the distortion of linear channel. Linear equalizer can eliminate the influence of ISI and improve the correct rate of receiv-ing character. When the equalizer tap interval is the symbol interval T (also called Baud), it is called the Baud spaced equalizer(BSE). However, there are some disadvantages in Baud spaced equalizer, and the per-formance is not good. In contrast, the equalizer with a fractional ratio of Baud interval is superior to Baud spaced equalizer, and it is called the fractionally spaced equalizer(FSE). 


\section{Simulation Model}

Set the a(n) for the launch of the $\mathrm{N}$ moment, $\mathrm{x}(\mathrm{n})$ for the $\mathrm{N}$ moment equalizer input, ck(n) for the $\mathrm{N}$ moment equalizer tap coefficient, $\hat{y}(n)$ for the $\mathrm{N}$ moment equalizer output.

Godard constant is

$$
R_{2}=\frac{E\left\{|a(n)|^{4}\right\}}{E\left\{|a(n)|^{2}\right\}} \text {. }
$$

Cost function is

$$
J_{C M}=\frac{1}{4} E\left\{\left(R_{2}-\left|y_{n}\right|^{2}\right)^{2}\right\} \text {. }
$$

True gradient is

$$
\nabla\left(J_{C M}\right)=y_{n} E\left\{R_{2}-\left|y_{n}\right|^{2}\right\} \text {. }
$$

Stochastic gradient(error) is

$$
e(n)=\hat{\nabla}\left(J_{C M}\right)=y_{n}\left(R_{2}-\left|y_{n}\right|^{2}\right) \text {. }
$$

The blind Baud spaced equalizer using the constant modulus algorithm can be expressed as follows:

Equalizer:

$$
\hat{y}(n)=\sum_{k=-L}^{L} c_{k}^{*}(n-1) x(n-k) \text {. }
$$

Error:

$$
e(n)=y(n)\left[R_{2}-|y(n)|^{2}\right] \text {. }
$$

Update:

$$
\mathbf{c}(n)=\mathbf{c}(n-1)+\mu \mathbf{x}(n) e^{*}(n) \text {. }
$$

Godard constant:

$$
R_{2}=\frac{E\left\{|a(n)|^{4}\right\}}{E\left\{|a(n)|^{2}\right\}}
$$

The blind fractionally spaced equalizer using the constant modulus algorithm can be expressed as follows:

Equalizer:

$$
\begin{aligned}
\hat{y}(n) & =\sum_{k=0}^{M-1} c_{k}^{\text {even }}(n-1) x^{\text {odd }}(n-k)+\sum_{k=0}^{M-1} c_{k}^{\text {odd }}(n-1) x^{\text {even }}(n-k) \\
& =\mathbf{c}^{T}(n-1) \mathbf{x}(n) .
\end{aligned}
$$

Error, update formula and Godard constant are the same as above.

\section{Simulation Result}

16-QAM data transmission system is used in the simulation.

Additive White Gauss Channel. Channel 1: additive white Gauss noise channel, where the channel transfer function is $H(z)=0.2+0.5 z^{-1}+z^{-2}-0.1 z^{-3}$, and the signal to noise ratio SNR is $20 \mathrm{~dB}$.

The change of the error is more chaotic when the DB unit is used, and the change rule can be seen by the general coordinate.

(1) The blind Baud spaced equalizer using the constant modulus algorithm

Equalizer order $\mathrm{L}$ is 20 , and tracking speed $\mu$ is 0.001 . 3 Simulation results are show as follows. 


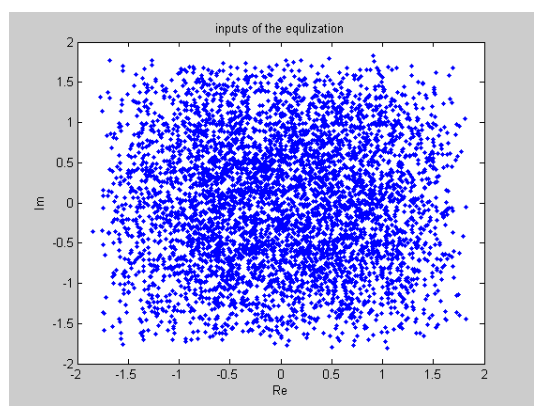

(a) inputs

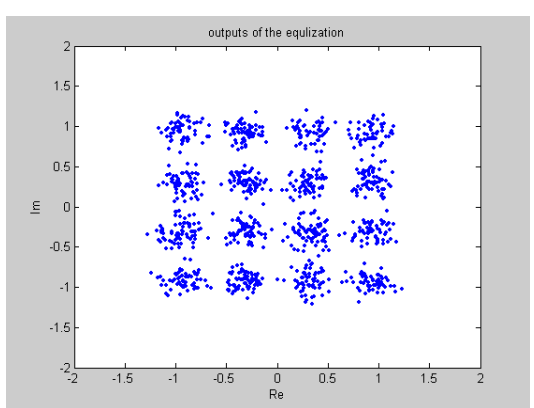

(b) outputs

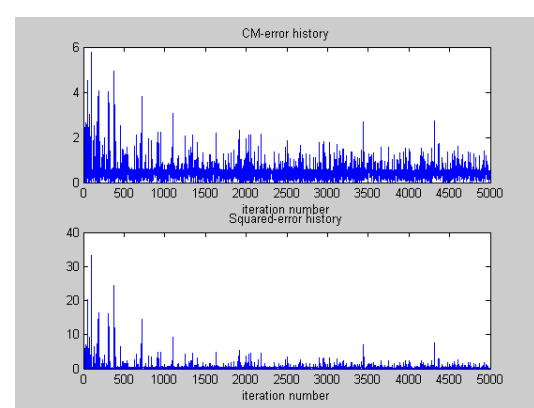

(c) CM-error history

Figure 1. Simulation result 1

(2) The blind fractionally spaced equalizer using the constant modulus algorithm

Equalizer order $2 \times \mathrm{M}$ is 16 , and tracking speed $\mu$ is 0.001 . Simulation results are show as follows.

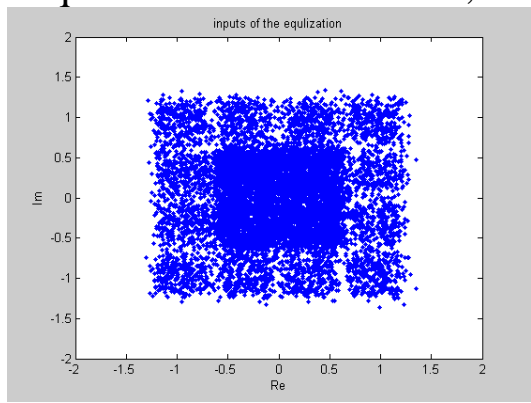

(a) inputs

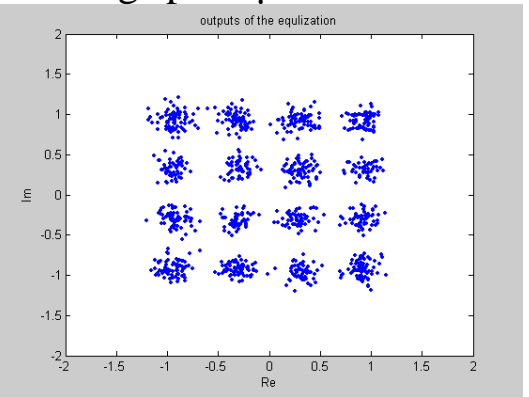

(b) outputs

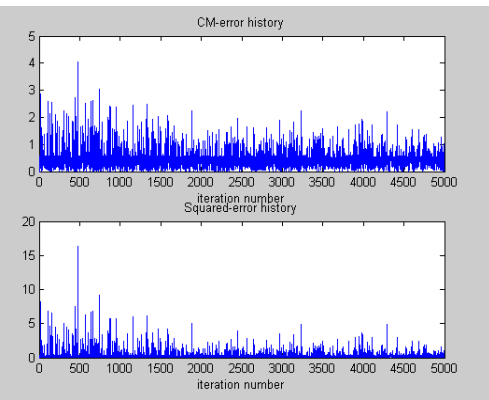

(c) CM-error history

Figure 2. Simulation result 2

The simulation results show that under the condition of fixed channel parameters, the adaptive constant modulus algorithm can achieve a good performance of blind equalization, and restore the constellation of the transmitter. Using adaptive algorithm, the CM error decreases with the number of iterations, and the performance is getting better and better.

Changing the tracking speed $\mu$ will change the performance. The convergence speed is larger, and the stability is poorer, when $\mu$ is larger. Therefore, the appropriate tracking speed should be selected.

Comparison of two different methods of Baud spaced and fractionally spaced, from Figure 1(b) and Figure 2(b), we can see that the constellation of fractionally spaced equalizer is closer to the constellation of the transmitting character. The preformation of the transmitting character is better. And equalizer order of fractionally spaced equalizer is less than Baud spaced equalizer.

Rayleigh Fading Channel. Channel 2: Rayleigh fading channel, channel parameters are the same as the first simulation. Carrier frequency is $2 \mathrm{GHz}$, mobile symbol rate is $1.025 \times 105$, Doppler spectrum $C$ is $[0,-3,-10,-15] \mathrm{dB}$, and 4 unrelated path, $\mathrm{SNR}=20 \mathrm{~dB}$.

(1) The blind Baud spaced equalizer using the constant modulus algorithm

Equalizer order L is 20 .

A. Speed of mobile station $\mathrm{v}=40 \mathrm{~m} / \mathrm{s}$

Simulation results are show as follows.

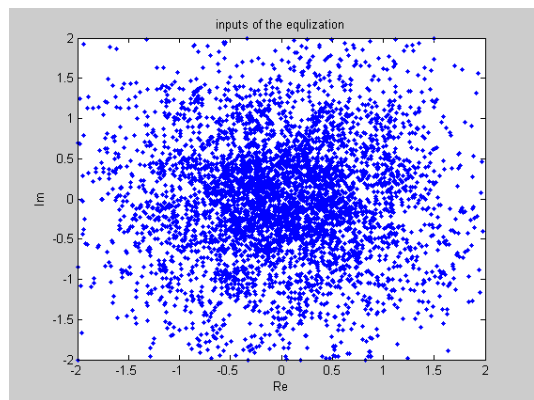

(a) inputs

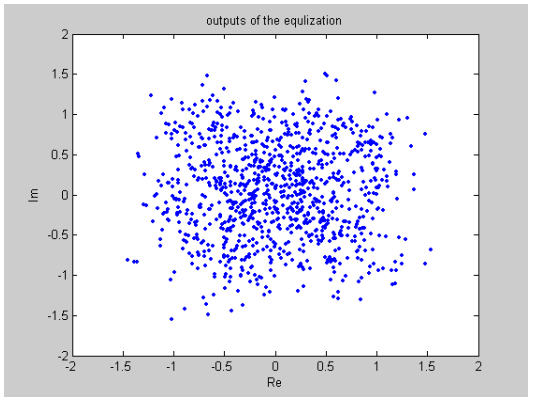

(b) outputs

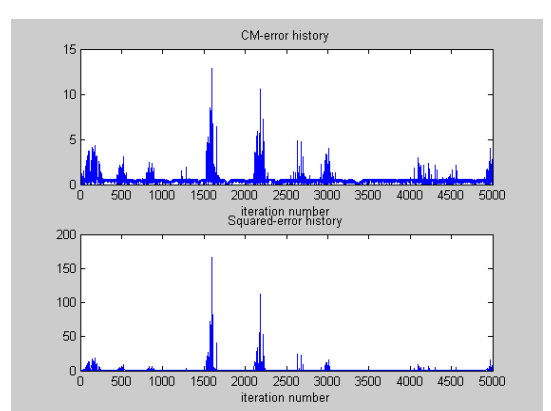

(c) CM-error history

Figure 3. Simulation result 3 
B. Speed of mobile station $\mathrm{v}=5 \mathrm{~m} / \mathrm{s}$

Simulation results are show as follows.

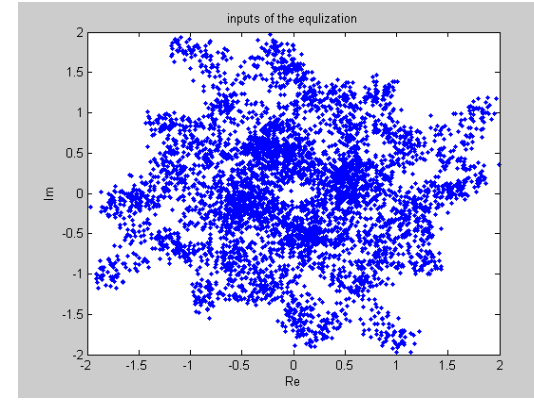

(a) inputs

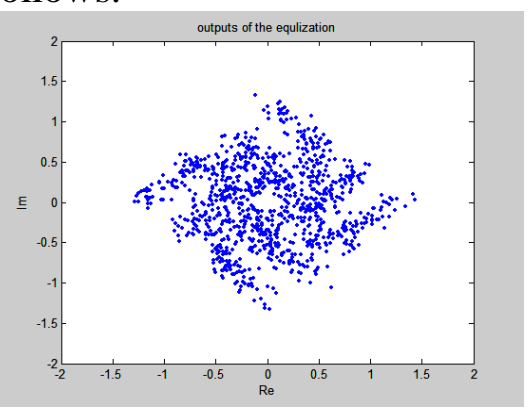

(b) outputs

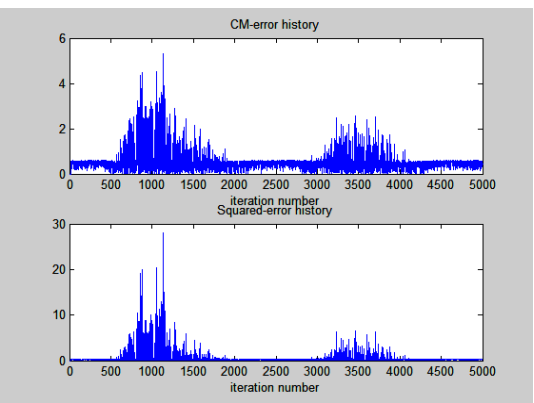

(c) CM-error history

Figure 4. Simulation result 4

(2) The blind fractionally spaced equalizer using the constant modulus algorithm Equalizer order $2 \times \mathrm{M}$ is 16 .

A. Speed of mobile station $\mathrm{v}=40 \mathrm{~m} / \mathrm{s}$

Simulation results are show as follows.

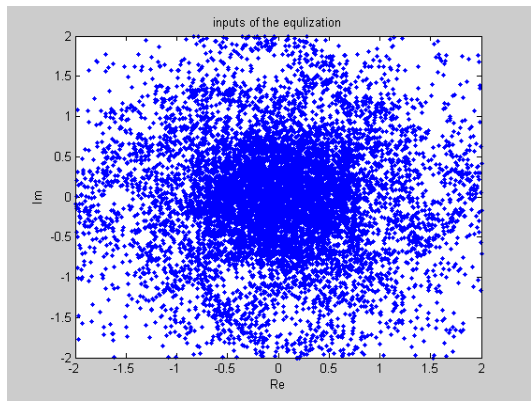

(a) inputs

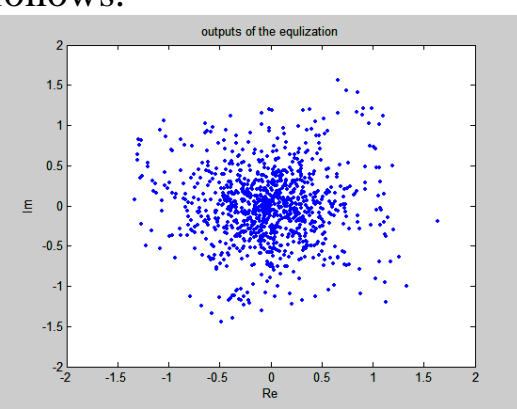

(b) outputs

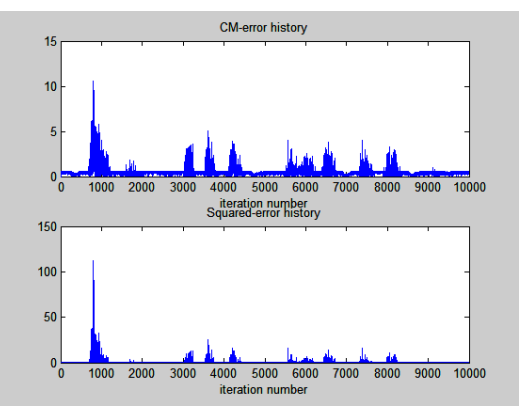

(c) CM-error history

Figure 5. Simulation result 5

B. Speed of mobile station $\mathrm{v}=1 \mathrm{~m} / \mathrm{s}$

Simulation results are show as follows.

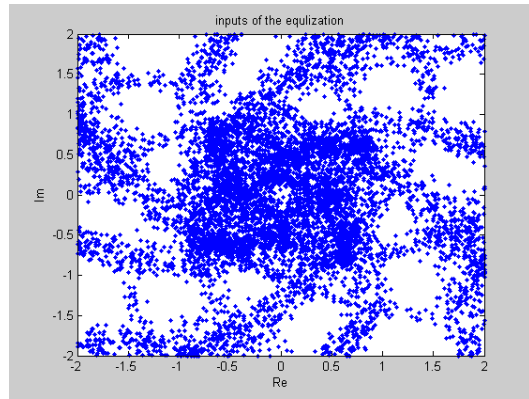

(a) inputs

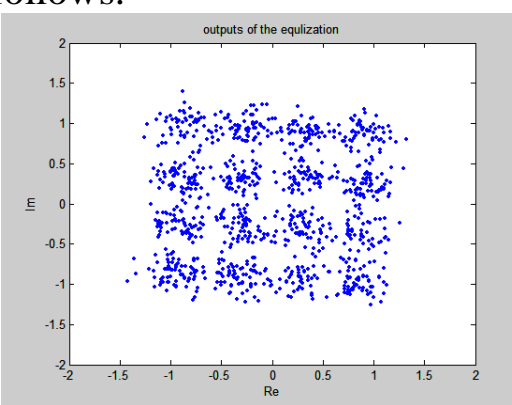

(b) outputs

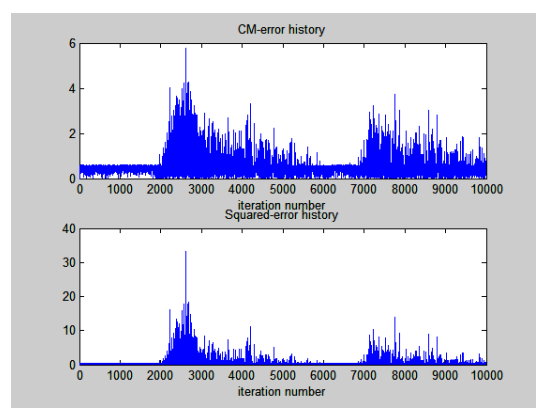

(c) CM-error history

Figure 6. Simulation result 6

Rayleigh channel is fast fading, and it changes quickly with time. The greater the moving speed of the mobile station, the greater the change of channel characteristics. In the case of high-speed movement of the mobile station $(\mathrm{v}=40 \mathrm{~m} / \mathrm{s})$, the channel characteristics change very quickly. From Figure 3(c) and figure 5(c) of the error curve, whether it is Baud spaced or fractionally spaced equalizer, adaptive algorithm cannot track changes in channel characteristics. From the equalizer output constellation figure 3(b) and figure 5(b), whose output is out of order, not to restore the input character constellation, and it is difficult to restore the input signal.

When the speed of the mobile station is low $(\mathrm{v}=5 \mathrm{~m} / \mathrm{s})$, the channel characteristic change speed is reduced. The adaptive constant modulus algorithm can track the change of channel characteristics from 
the error curve of figure 4(c) and Figure 6(c). From the equalizer output constellation Figure 4(b) and Figure 6(b), the output is much better than the high-speed movement, constellation diagram can see the shape of the input constellation, but the effect is still relatively poor.

\section{Conclusion}

When the channel characteristics are unchanged, the adaptive constant modulus algorithm can achieve blind equalization. Quicker the channel characteristics change, worse the tracking ability of adaptive constant modulus algorithm is getting.

The fractionally spaced equalizer avoids the aliasing caused by under sampling and compensates for the channel distortion in the received signal. The performance of fractionally interval sampling is better than baud interval sampling.

\section{References}

[1] Johnson R. et.al. Blind equalization using the constant modulus criterion: A review[J]. Proc. IEEE, vol.86, no.10, pp.1927-1949, 1998.

[2] James P. LeBlanc, Inbar Fijalkow, C. Richard Johnson. CMA fractionally spaced equalizers: Stationary points and stability under i.i.d. and temporally correlated sources[J]. Int. J. Adapt. Control Signal Process, 1998(2).

[3] D.R. Srinivas, K.E. Sreenivasa Murthy. Blind Adaptive Equalization of Complex Signals based on the Constant Modulus Algorithm[J]. International Journal of Computer Applications. 2010(5).

[4] Tai Wu Xia,Hong Jun Liu. Multiuser Beamforming Using Weighted Orthogonal Constant Modulus Algorithm[J]. Advanced Materials Research. 2011(204). 